\title{
Design and testing of a DSS to help reduce bias in judgemental forecasting
}

\author{
Michael Lawrence \\ School of Information Systems \\ University of New South Wales \\ Sydney 2052 Australia \\ Telephone: (02)385.4417 Fax: (02)662.4061 \\ e-mail: M.Lawrence@unsw.edu.au
}

\begin{abstract}
One of the most widely occurring management decision tasks is preparing the monthly forecasts. This execution of this task requires the application of management judgement to combine contextual knowledge with the time series historical data. The task would appear well suited to the application of a DSS. Yet surveys in the USA, UK and Australia show very low penetration of any direct computer support in the estimation of forecasts. This paper gives some clues as to the reasons for this state of affairs and explores a tool, potentially more acceptable to forecasters which is designed to help reduce the human bias present in extrapolating a time series. The tool which is behaviourally rather than mathematically based, is shown to improve estimation accuracy over a purely judgemental alternative and to be significantly better than the naive forecast.
\end{abstract}

Forecasting, decision support, DSS, judgement, bias

\section{Keywords}

Forecasting, decision support, DSS, judgement, bias

\section{INTRODUCTION}

Surveys of forecasting practice in Australia, USA and the UK indicate the continuing high use of judgemental and opinion based methods in preference to quantitative methods (e.g. Sparkes and McHugh, 1984; Dalrymple, 1987; Tarranto, 1989; Sanders and Manrodt, 1994). These studies of business (mostly sales) forecasting practice reveal that only around $10 \%$ of the firms surveyed use quantitatively based forecasting techniques and that the number of firms who have tried and subsequently abandoned these techniques is about double the number currently using them. This situation cannot be blamed on lack of supply of forecasting technology there has been an enthusiastic response by system developers and there is a wide range of 
inexpensive and user-friendly PC windows and main-frame based software to choose from. These systems benefit from the great volume of forecasting research conducted over the last 25 years. As might be expected, this lack of use has been a cause of concern to researchers and management educators who believe that valuable technology is remaining under-utilised (Makridakis, 1988; Armstrong, 1994).

Improvements in forecasting could have a significant impact on business. Most businesses are involved in regularly developing forecasts and losses through inaccurate forecasts can be high (Makridakis, 1988). For example, organisations involved in warehousing and distribution incur holding and obsolescence costs on their inventory which can be around $25 \%$ per annum. In addition lost business from stockouts may be even more damaging. But the preference revealed in these studies for judgemental methods cannot be dismissed as the action of a business community unconcerned by forecast errors. The surveys cited show that business does keep records of forecast accuracy and that they are unlikely to continue to use a method which does not perform (Dalrymple, 1988). Nor can the preference be attributed to lack of knowledge of quantitative forecasting technology. Armstrong (1994) comments that an increasing exposure to forecasting technology has not increased its use.

The barriers to information systems implementation success theorised and explored in the literature include (Hirschheim and Newman, 1988):

User reasons (e.g. lack of felt need, conservatism),

technology and implementation reasons (e.g. poor system quality, inappropriate design, poor user training), and

organisational reasons (e.g. inadequate management support).

Before we apply this model to computer based forecasting systems we need to briefly present the current paradigm which structures and underpins forecasting research and software products. The current paradigm of forecasting research and software products decomposes the forecasting task into the following steps

a) Develop an extrapolation on the basis of the time series data.

b) If necessary, modify the extrapolation using contextual data. This contextual data may reflect marketing plans, macroeconomic expectations, competitor intelligence or microeconomic factors such as labour disturbances.

Forecasting research for the last fifteen years has been dominated by the pursuit of step (a) above with the search for the best forecast model that generates the most accurate extrapolation. This activity has lead to a number of laboratory based forecasting competitions (e.g. Makridakis et al, 1982; Makridakis et al, 1993) which have acted as a significant focus for further research. However there is not universal acceptance of step (b) with some authors cautioning against modifying the extrapolation except under the most exceptional circumstances such as what are called "broken leg cues" (Armstrong, 1985). Furthermore step (b) is often difficult to perform because it may not be clear the extent to which recent exceptional phenomena have been incorporated into the quantitative forecast or have been left out (being treated as a random event) and therefore need to be incorporated judgementally. 
These problems with step (b) have generated the feeling that the forecast should not be manually modified, leading to the situation where the forecasting system replaces rather than supports the business forecaster. We thus suggest that an important reason for the lack of use of forecasting technology is its inappropriate design and the lack of appeal of its underlying paradigm.

An alternative paradigm for forecasting was pioneered by Edmundson (1990) with the DSS tool GRAFFECT, later incorporated into some commercial forecasting systems (e.g. foreGraph). These systems are not based on the two steps given above nor are they based on a fitting a mathematical model to the time series history. Rather they allow the forecaster to develop the forecast judgementally. The task decomposition used in these tools breaks the time series into its trend, seasonal and randomness elements. The forecasting user, sitting before a graphical representation of the series on the computer screen, has a choice of various forms of statistical support and also can judgementally intervene to modify or dictate the seasonal and the trend terms. As seasonals and trend are generally far from stationary, this is often a very desirable facility. The forecast is then made on the de-trended and deseasonalised series either judgementally or using a quantitative forecast model. Edmundson (1990) showed that the judgemental forecast made using his GRAFFECT tool was more accurate than that made using a pencil and paper judgemental approach and was also more accurate than deseasonalised exponential smoothing, the most accurate quantitative method for the time series used in his experiment.

However there would appear to be further gains in accuracy possible. Lawrence and O'Connor (1995) showed that a judgemental extrapolation was subject to a number of biases which reduce its accuracy. Their experiment suggested that subjects forecasting a time series tended to be too extreme in their estimates. This result can also be viewed as a reversal of the usual anchor and adjustment bias in which subjects anchor on a value already known and arrive at their estimate through making an adjustment (generally too small) from this anchor point (Tversky and Kahneman, 1974). In the case of time series forecasting, subjects appear to adjust too much from the anchor point of the most recent observation. Harvey (1995) also observed this same phenomenon and argued it may be due to the subjects seeking to estimate a forecast which "looked like" the original observed series in both its trend and its randomness. Lawrence and O'Connor (1995) argued it may be due to the subjects not discounting their estimates for the uncertainty of the direction of the movement from the anchor point. They demonstrated that if the error in picking the right direction (up or down) was taken as the uncertainty, and the amount of adjustment discounted by the uncertainty, then the estimates were effectively debiased. Thus if this approach could be incorporated into the procedure, it would appear that the resulting forecast could be improved. This paper examines this approach to improving the estimation of a judgemental forecast when the time series has been deseasonalised. 


\section{RESEARCH QUESTIONS}

In a laboratory setting Lawrence and O'Connor (1995) found that subjects were biased in their forecast estimates by adjusting too far from the anchor point (taken to be the last observation). Lawrence and O'Connor suggested that subjects employed a two stage process for estimating their forecast. In the first stage the direction of change was picked and in the second, the size of the adjustment from the anchor point was estimated. They argued that the subjects did not reflect in the second stage the likelihood of error in the first stage. When the subjects' results were discounted for their probability $p$ of picking the correct direction of the change (from the last observation) the forecast accuracy was significantly improved. The discounted adjustment $D A D J$ can be shown (Lawrence and O'Connor, 1995) to be given by the formula:

$D A D J=(2 p-1) \cdot A D J$

where $A D J$ is the adjustment from the anchor point in which no allowance has been made for uncertainty in the direction in the adjustment. One can see if the probability $p$ is 0.5 (indicating no belief about whether the time series will go up or down) that $D A D J=0$, while if $p=1$, (perfect confidence) then $D A D J=A D J$. In this paper a DSS was developed and tested which decomposed the task into the two stages and discounted the size of the adjustment for the ambiguity of picking the direction. Such a DSS would be able to be incorporated into a tool like GRAFFECT to improve the core extrapolation task.

\section{RESEARCH METHODOLOGY}

A DSS was developed which presented a graph of a time series to a subject and asked that a one step ahead forecast be developed in two stages:

Stage 1. Estimate the direction of the change from the last observation and the likelihood of the chosen direction being correct (only the rounded percentages $50 \%, 60 \%, 70 \% \ldots 100 \%$ were available for choice).

Stage 2. Estimate the amount of change from the last observation conditional on the direction being correct.

Thus the major feature of this DSS is the structuring of the task and the detailed decomposition of the estimate into the above components.

The subject had presented to him on the computer screen a graph showing the time series at an appropriate presentation scale (Lawrence and O'Connor, 1993). Via a mouse and the numeric keypad the subject entered the information shown above following definitions of what to enter displayed on the screen. The screen then displayed both the initial estimate (i.e. undiscounted), the discounted estimate and after the subject indicated he was satisfied with the estimates in that round, the actual was displayed. Colour was used to differentiate these different data points. The cycle was then repeated with each subject estimating 24 forecasts for 
the one time series. A total of 30 time series were used in the experiment. These time series were drawn randomly from the real-life monthly series in the M-Competition (Makridakis et al., 1982) and deseasonalised using the same seasonal factors as used in the M-Competition. Feedback was provided to subjects by the following means: (a) screen display of the original and discounted estimates and the actual value; (b) the progressive score of the percent correct for direction of change.

A reference group of subjects estimated the forecasts without the benefit of the decomposition based DSS using instead a computer presentation of the time series graph similar to that in the DSS. The subject indicated his estimate of the forecast using a mouse to position a cross hair on the screen.

The subjects were undergraduate and graduate students at the University of New South Wales majoring in Information Systems. No monetary or other compensation was paid to the subjects.

\section{ANALYSIS METHODOLOGY}

The metric used to assess forecast accuracy and compare the DSS with the reference was the Mean Absolute Percentage Error (MAPE), defined as the mean of APE where

$\mathrm{APE}=|\mathrm{ACTUAL}-\mathrm{FORECAST}| / \mathrm{ACTUAL}$

This metric was selected for several reasons. First it is less affected than squared measures by extreme errors, and thus is a good measure for comparisons across techniques. Second, the metric is independent of scale, enabling a comparison to be made across a number of different time series. Perhaps most importantly it is the dominant metric used in practice to measure forecast accuracy.

In addition to the reference forecast of subjects using the simple (non-decomposition) graph based DSS, the forecast accuracy of a naive forecast was calculated. The naive forecast (the last actual) has been shown to be a surprisingly accurate forecast and has been often used as a reference to measure forecast accuracy (e.g. Lawrence and O'Connor, 1992).

\section{RESULTS}

The MAPE accuracy results for the DSS group are shown in Figure 1 along with the results for the reference group (called Graph) and the naive forecasts. When paired t-tests were run, the DSS accuracy was better than both Graph and Naive above the 0.06 significance level. Naive and Graph were not significantly different in accuracy as was expected from the results reported in Lawrence, Edmundson and O'Connor (1985). Thus while the differences in accuracy between the DSS and the reference group may not appear to be exceptional they are nevertheless significant and suggest an important direction in improving forecast accuracy. 


\section{MAPE}



Figure 1. Comparison of DSS, Graph and Naive forecast accuracy

\section{DISCUSSION}

A more detailed examination of the results indicated that although the DSS users did exhibit superior performance, they were not learning from their experience and improving their estimation skills. When the 24 months of each subject's trials were split into two groups (months 37-48 and months 49-60), there was no improvement in estimating the probability of picking the direction of change. The usual metric for judging the accuracy of a subjective probability estimate is the calibration of the estimate in the light of subsequent events. The results in Table 1 below give the average expectation of being correct in the chosen direction and the actual percent correct. This shows that the expectation was about $10 \%$ higher than the actual realisation indicating overconfidence in the judgements. Furthermore the overconfidence was not adjusted in the light of the worse than expected performance despite a display which presented as a running score the percentages for picking correctly the direction of change.

\section{Expected Actual}

\begin{tabular}{|c|c|}
\hline Months 37-48 & $65.4 \%$ \\
\hline
\end{tabular}

$\begin{array}{lll}\text { Months } 49-60 & 65.6 \% & 55.6 \%\end{array}$

Table 1. Expected and Actual probability of forecasted direction.

If the confidence in the direction had been correctly estimated the performance of the DSS users would have been even better. This opens the question as to how the forecasters can be helped through a modification to the DSS to improve their estimation skills. We see more 
vividly the calibration errors when the calibration diagram presented in Figure 2 is inspected. This diagram presents for each stated confidence level the actual percentage of times the chosen direction was correct and the number of estimates (n) falling into that interval. For example when the 41 subject estimates claimed $100 \%$ confidence they were correct $75.6 \%$ of the time. Apart from at the $50 \%$ level, (for which there could hardly be any overconfidence) they were consistently overconfident. The worst result is at the $80 \%$ stated confidence level where only $50.1 \%$ of the direction predictions were correct. This general overconfidence has been observed in numerous studies in other task areas (Tversky and Kahneman, 1974) but rarely is the calibration curve so flat as in Figure 2. This figure reveals little skill in correctly estimating confidence and indicates substantial opportunity for improving performance.

\section{Calibration of Direction Prediction Confidence}

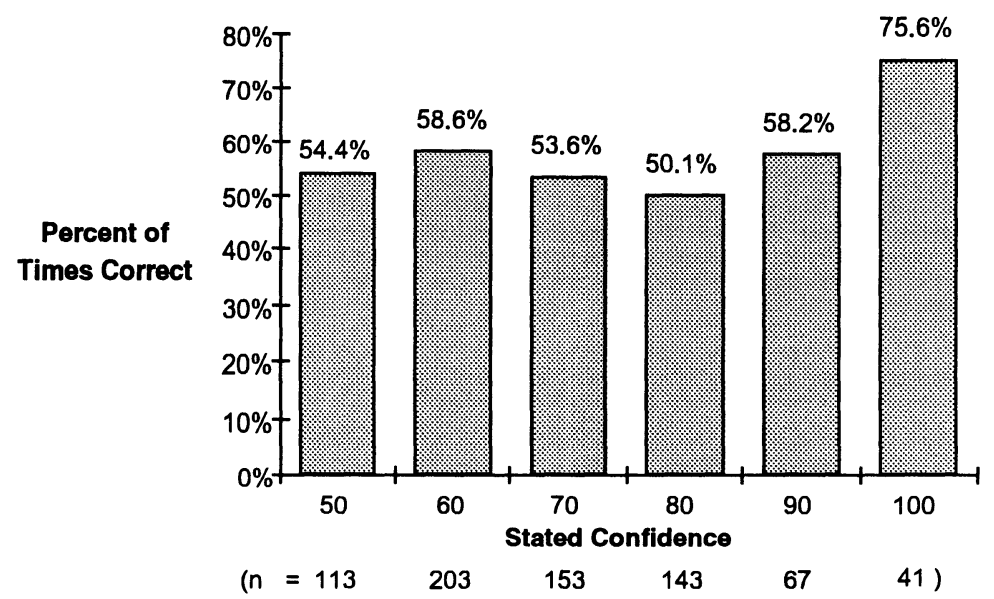

Figure 2 Calibration of direction prediction confidence

\section{CONCLUSIONS}

This study has suggested that the reason for the low penetration of computer based support for forecasting is the lack of user acceptance of a paradigm which places too much emphasis on the extrapolation of the time series and too little on the judgement and expertise of the forecasters. A new paradigm focused on supporting the judgements of the forecasters in all stages of the forecast preparation was introduced and the problem of reducing persistent human bias in extrapolation was defined as the task of this study. Based on earlier work by the author a two stage forecast decomposition embedded in a DSS was used to improve human judgement. This was shown to be significantly better than unaided judgement. However there 
was seen to be still a significant overconfidence bias in the judgements which suggests there is considerable further scope for improvement in the process. This work demonstrates a behaviourally based DSS in contrast to the usual mathematically based tools.

\section{REFERENCES}

Armstrong, J.S. (1985) Long Range Forecasting: From crystal ball to computers. 2nd Edition. Wiley, New York.

Armstrong, J.S. (1994) Review of Sanders and Manrodt. International Journal of Forecasting, 10, 471-472.

Dalrymple, D. J. (1987) Sales forecasting practices: Results from a US survey. Journal of Forecasting 3, 379-391.

Edmundson R. H, Lawrence M. J. \& O'Connor M. J. (1988) The use of non-time series data in sales forecasting: a case study. Journal of Forecasting, 7, 201-212.

Edmundson, R. (1990) Decomposition: a strategy for judgemental forecasting. Journal of Forecasting, 4, 305-314.

Harvey, N. (1995) Why are judgements less consistent in less predictable task situations? Organisational Behaviour and Human Decision Processes, 63, 247-263.

Hirschheim, R. \& Newman, M. (1988) Information systems and user resistance: theory and practice. The Computer Journal, 31, 398-408.

Lawrence, M. J. and O'Connor, M. J.(1995) The anchor and adjustment heuristic in time series forecasting. Journal of Forecasting, 14, 443-451.

Lawrence, M. J. and O'Connor, M. J. (1992) Exploring judgemental forecasting. International Journal of Forecasting, 8, 15-26.

Lawrence, M. J. and O'Connor, M. J. (1993) Scale, randomness and the calibration of judgemental confidence intervals", Organisational Behaviour and Human Decision Processes, 56, 441-458.

Lawrence, M. J. Edmundson, R. H. and O'Connor, M. J. (1985) An examination of the accuracy of judgemental extrapolation of time series, International Journal of Forecasting, 1, 25-35.

Makridakis, S, Anderson, A, Carbone, R, Fildes, R, Hibon, M, Lewandowski, R, Newton, J, Parzen, E, and Winkler, $R,(1982)$ The accuracy of extrapolation (time series) methods: results of a forecasting competition. Journal of Forecasting, 1, 111-153.

Makridakis, S, Chatfield, C, Hibon, M, Lawrence, M, Mills, T, Ord, K, and Simmons, L, (1993) The M2-Competition: A real time judgementally based forecasting study, International Journal of Forecasting, 9, 5-22.

Makridakis, S, (1988) Metaforecasting International Journal of Forecasting 4, 467-491.

Sanders, N, and Manrodt, K. (1994) Forecasting practices in US corporations: survey results, Interfaces, 24, 92-100.

Sparkes, J. R. and McHugh, A. K. (1984) Awareness and use of forecasting techniques in British industry, Journal of Forecasting, 3, 37-42.

Tarranto, M. (1989) Sales forecasting practice in Australia. Unpublished B Com honours thesis. University of NSW, Kensington NSW.

Tversky, A and Kahneman, D (1974) Judgement under uncertainty: Heuristics and Biases Science, 185, 1124-1131. 


\section{BIOGRAPHY}

Michael Lawrence is Professor of Information Systems at the University of New South Wales, Australia. His major area of research is support for managerial decision making where judgement and quantitative analysis need to both play a part. He has a particular interest in time series forecasting and his publications include Management Science, International Journal of Forecasting, Organisational Behaviour and Human Decision Processes, MIS Quarterly and the Journal of Forecasting. 